\title{
The impact of histology and delivered dose on local control of spinal metastases treated with stereotactic radiosurgery
}

\author{
Yoshiya Yamada, MD, FRCPC, ${ }^{1}$ Evangelia Katsoulakis, MD, ${ }^{1}$ Ilya Laufer, MD, ${ }^{2}$ \\ Michael Lovelock, PhD, ${ }^{4}$ Ori Barzilai, MD, ${ }^{2}$ Lily A. McLaughlin, BS, ${ }^{2}$ Zhigang Zhang, PhD, ${ }^{5}$ \\ Adam M. Schmitt, MD, ${ }^{1}$ Daniel S. Higginson, MD, ${ }^{1}$ Eric Lis, MD, ${ }^{3}$ Michael J. Zelefsky, MD, ${ }^{1}$ \\ James Mechalakos, $\mathrm{PhD},{ }^{4}$ and Mark H. Bilsky, MD ${ }^{2}$
}

Departments of ${ }^{1}$ Radiation Oncology, ${ }^{2}$ Neurological Surgery, ${ }^{3}$ Radiology, ${ }^{4}$ Medical Physics, and ${ }^{5}$ Epidemiology and Biostatistics, Memorial Sloan Kettering Cancer Center, New York, New York

OBJECTIVE An analysis of factors contributing to durable radiographic control of spinal metastases was undertaken, drawing from a large single-institution database in an attempt to elucidate indications and dose requirements for successful treatment.

METHODS All patients treated at a single institution with stereotactic radiosurgery (SRS) of the spine as first-line therapy were assessed for local progression of the treated site, defined as radiographic enlargement of the treated tumor and/or biopsy-proven evidence of active tumor cells. All patients were followed with CT, PET, or MR imaging every 3-6 months until death. Treatment decisions were made by a multidisciplinary team of radiation oncologists, neurosurgeons, and neuroradiologists. Target volumes were defined according to the international consensus guidelines and were reviewed in a multidisciplinary conference. Image-guided techniques and intensity modulation were used for every case. The tumor's histological type, gross tumor volume (GTV), dose that covers $95 \%$ of the GTV (GTV D95), percentage of GTV covered by $95 \%$ of the prescribed dose (GTV V95), planning target volume (PTV), dose that covers $95 \%$ of the PTV (PTV D95), and percentage of PTV covered by $95 \%$ of the prescribed dose (PTV V95) were analyzed for significance in relation to local control, based on time to local progression.

RESULTS A total of 811 lesions were treated in 657 patients between 2003 and 2015 at a single institution. The mean follow-up and overall survival for the entire cohort was 26.9 months (range 2-141 months). A total of 28 lesions progressed and the mean time to failure was 26 months (range 9.7-57 months). The median prescribed dose was 2400 cGy (range 1600-2600 cGy). Both GTV D95 and PTV D95 were highly significantly associated with local failure in univariate analysis, but GTV and PTV and histological type did not reach statistical significance. The median GTV D95 for the cohort equal to or above the GTV D95 1830 cGy cut point (high dose) was 2356 cGy, and it was 1709 cGy for the cohort of patients who received less than 1830 cGy (low dose). In terms of PTV D95, the median dose for those equal to or above the cut point of 1740 cGy (high dose) was 2233 cGy, versus 1644 cGy for those lesions below the PTV D95 cut point of 1740 cGy (low dose).

CONCLUSIONS High-dose single-session SRS provides durable long-term control, regardless of the histological findings or tumor size. In this analysis, the only significant factors predictive of local control were related to the actual dose of radiation given. Although the target volumes were well treated with the intended dose, those lesions irradiated to higher doses (median GTV D95 2356 cGy, minimum 1830 cGy) had a significantly higher probability of durable local control than those treated with lower doses (median PTV D95 2232 cGy, minimum of $1740 \mathrm{cGy}$ ) ( $p<0.001)$. Patients in the high-dose cohort had a $2 \%$ cumulative rate of local failure. Histological findings were not associated with local failure, suggesting that radioresistant histological types benefit in particular from radiosurgery. For patients with a favorable prognosis, a higher dose of SRS is important for long-term outcomes.

https://thejns.org/doi/abs/10.3171/2016.9.FOCUS16369

KEY WORDS stereotactic radiosurgery; local control; spinal metastases

ABBREVIATIONS CIF = cumulative incidence of local failure; CTV = clinical target volume; GTV = gross tumor volume; GTV D95 = dose that covers $95 \%$ of the GTV; GTV V95 = percentage of GTV covered by $95 \%$ of the prescribed dose; PTV = planning target volume; PTV D95 = dose that covers $95 \%$ of the PTV; PTV V95 = percentage of PTV covered by $95 \%$ of the prescribed dose; SRS = stereotactic radiosurgery.

SUBMITTED August 29, 2016. ACCEPTED September 20, 2016.

INCLUDE WHEN CITING DOI: 10.3171/2016.9.FOCUS16369. 
$\mathrm{S}$ TEREOTACTIC radiosurgery (SRS) in which image guidance is used to deliver a single high dose of radiation to spine tumors is rapidly gaining acceptance ${ }^{5,23}$ as an effective modality to manage this vexing and common oncological problem. However, the importance of the actual dose of radiation given, as well as the impact of tumor histological type on long-term outcomes after spine radiosurgery, is still a matter of debate. A wide range of doses has been reported for SRS, and the doses vary according to institutional prescribing practices, which makes interpretation of the literature difficult. Tumor histological type is recognized as an important factor in expected outcomes for conventional palliative radiotherapy for spinal metastases, but is thought to be less important in the case of high-dose SRS. This histology-independent response may be due to unique radiobiological mechanisms of action. ${ }^{9}$ The NOMS (neurological, oncological, mechanical, and systemic) framework ${ }^{14}$ and similar paradigms have been put forward to define the role of spine radiosurgery in the management of spinal metastases, using tumor histological findings and the ability to deliver a sufficient dose to the tumor as key points in the decision-making process. A detailed analysis of a large single-institution experience of SRS of the spine incorporating histological type, tumor size, and dose parameters was undertaken to assess the impact of key factors associated with durable tumor control with radiosurgery.

\section{Methods}

Between 2003 and 2015, a consecutive series of 811 solid spine tumors in 657 patients were treated with high-dose, single-session spine radiosurgery at a single institution. No patient had either previously received radiation therapy or undergone prior spine surgery at the site of interest. All lesions were assessed by a multidisciplinary team that consisted of radiation oncologists, neurosurgeons, and neuroradiologists. Every tumor histological type was pathologically confirmed with institutional review. All tumors were treated with the aid of image guidance, and treatment plans were developed with intensity-modulated techniques. Every patient was followed with cross-sectional imaging (predominantly MRI) and follow-up visits every 3-6 months, and 19 patients have been lost to follow-up. Local failure was defined as enlargement of the treated tumor on imaging studies or positive pathological findings after treatment (biopsy or surgical). All follow-up images were reviewed by neuroradiologists.

Unless it was contraindicated, all patients underwent CT myelography to define the spinal cord and cauda equina at the time of simulation; MRI fusion was used in the remaining few cases. The clinical target volume (CTV) and gross tumor volume (GTV) were drawn consistent with the International Consensus Guidelines ${ }^{4}$ and underwent multidisciplinary review. The planning target volume (PTV) was constructed with a 2- to 3-mm margin around the CTV. However, the CTV and PTV contours were never allowed to transgress the spinal cord or cauda equina contour. After a dose escalation experience between 2003 and 2004 (from 1600 cGy initially to 2400 cGy currently), the predominant prescription dose
TABLE 1. Tumor characteristics in 811 lesions treated between 2003 and 2015

\begin{tabular}{|c|c|c|c|}
\hline Characteristic & $\begin{array}{c}\text { Total } \\
\text { No. }\end{array}$ & $\begin{array}{l}\text { No. of Local } \\
\text { Failures }\end{array}$ & $\begin{array}{c}\text { Crude } \\
\text { Failure (\%) }\end{array}$ \\
\hline \multicolumn{4}{|l|}{ Treated levels } \\
\hline Cervical & 85 & 4 & 5 \\
\hline Thoracic & 391 & 13 & 3 \\
\hline Lumbar & 258 & 7 & 3 \\
\hline Sacrum & 77 & 4 & 5 \\
\hline \multicolumn{4}{|l|}{ Histological findings } \\
\hline \multicolumn{4}{|l|}{ Radiosensitive } \\
\hline Breast & 32 & 1 & 3 \\
\hline Cervical & 10 & 0 & 0 \\
\hline Prostate & 91 & 5 & 6 \\
\hline Uterine & 13 & 1 & 8 \\
\hline Total & 146 & 7 & 5 \\
\hline \multicolumn{4}{|l|}{ Radioresistant } \\
\hline Renal cell carcinoma & 170 & 5 & 1 \\
\hline Sarcoma & 113 & 3 & 3 \\
\hline Thyroid cancer & 54 & 2 & 4 \\
\hline Chordoma & 32 & 3 & 9 \\
\hline Colorectal & 71 & 1 & 1 \\
\hline Esophageal & 25 & 1 & 4 \\
\hline Hepatobiliary & 27 & 0 & 0 \\
\hline Adenoid cystic head \& neck & 17 & 1 & 6 \\
\hline Non-small cell lung carcinoma & 102 & 1 & 1 \\
\hline Melanoma & 48 & 3 & 6 \\
\hline Nonseminomatous & 6 & 1 & 17 \\
\hline Total & 665 & 21 & 3 \\
\hline All & 811 & 28 & 3 \\
\hline
\end{tabular}

has been $2400 \mathrm{cGy}$. The spinal cord was constrained to a maximum dose of 1200-1400 cGy, and the cauda equina was constrained to 1600-1800 cGy. Inverse treatment planning algorithms were used to create highly conformal dose distributions, and doses were prescribed to the isodose line that provided optimal target coverage and then normalized to $100 \%$. Patients were treated after being placed supine in custom immobilization, which consists of molded alpha cradles with lateral support paddles, with the addition of a customized mask for lesions at T-5 and above, as has been previously described. ${ }^{15}$ All patients were treated on LINAC-based systems, initially with implanted fiducials and electronic portal imaging, or conebeam CT-based image guidance (since 2005).

Survival times and time to local failure were calculated from the date of treatment. Tumor histological type, GTV, the dose that covers 95\% of the GTV (GTV D95), the percentage of GTV covered by $95 \%$ of the prescribed dose (GTV V95), PTV, the dose that covers $95 \%$ of the PTV (PTV D95), and the percentage of PTV covered by $95 \%$ of the prescribed dose (PTV V95) were included in the analysis.

The primary end point for the analysis was time to local failure. Death without recurrence was regarded as a competing risk. The Fine-Gray competing risks model ${ }^{6}$ 


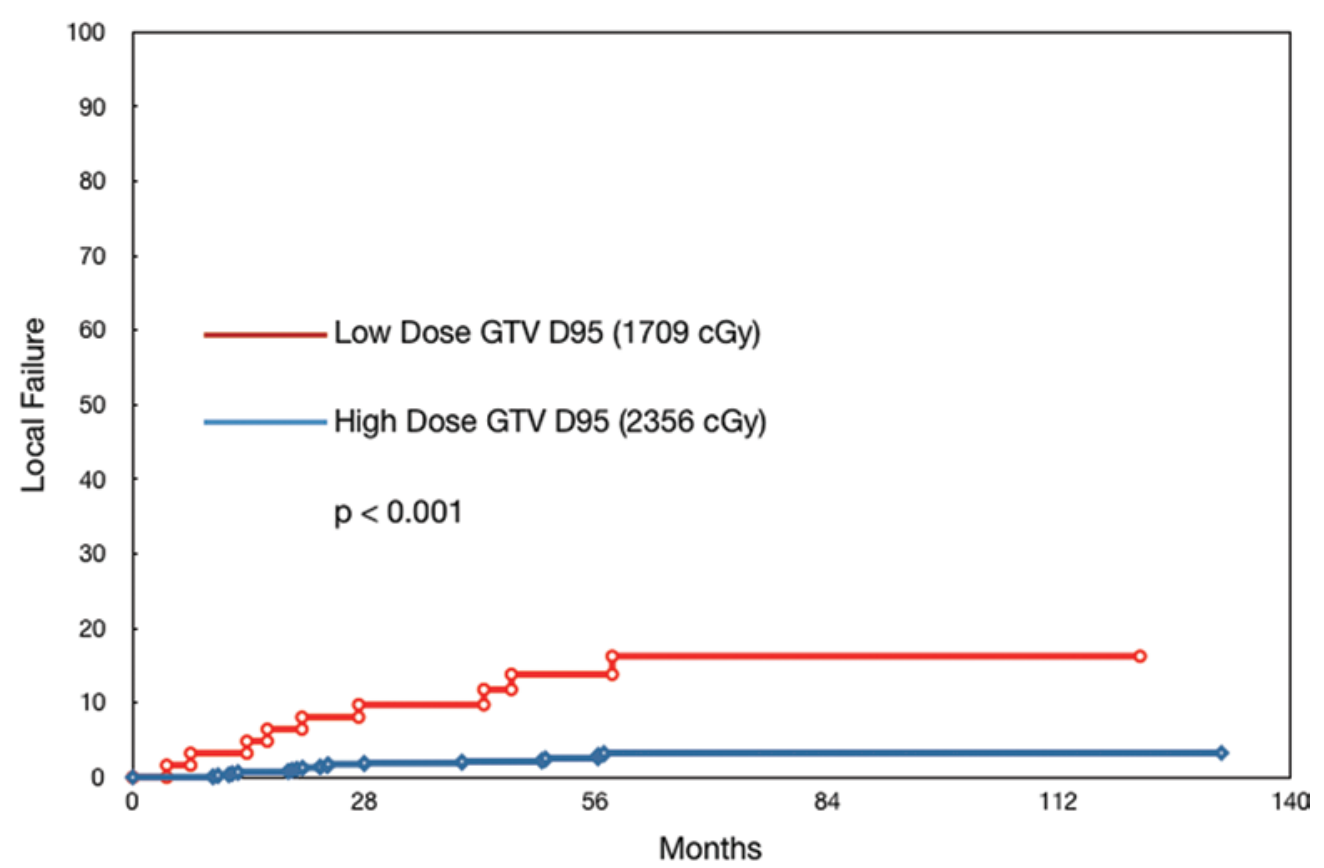

\begin{tabular}{|l|l|l|l|l|}
\hline CIF\% (95\%CI) & 12 months & 24 months & 36 months & 48 months \\
\hline Overall & $0.63 \%(0.08-1.2 \%)$ & $2.3 \%(1.2-3.3 \%)$ & $2.6 \%(1.2-3.7 \%)$ & $3.1 \%(1.8-4.4 \%)$ \\
\hline GTV D95 Low Dose & $3.2 \%(0-7.7 \%)$ & $8.1 \%(1.2-15 \%)$ & $9.7 \%(2.2-17 \%)$ & $14 \%(4.7-23 \%)$ \\
\hline GTV D95 High Dose & $0.42 \%(0-0.9 \%)$ & $1.8 \%(0.77-2.8 \%)$ & $1.9 \%(0.89-3 \%)$ & $2.1 \%(1-3.2 \%)$ \\
\hline
\end{tabular}

FIG. 1. Upper: Local control for GTV D95 cut point of 1830 cGy. The high-dose cohort median dose is $2356 \mathrm{cGy}$, and the lowdose cohort median dose is 1709 cGy. Lower: Chart showing the CIF.

was used for deriving the hazard ratio, and the Gray test was used for comparing cumulative incidence functions. ${ }^{12}$ Dose, tumor size (GTV), PTV, GTV V95, and PTV V95 values were analyzed as continuous variables. An optimal cut point for GTV D95 and PTV D95 values was determined based on time to local failure and was identified as the dose at which the smallest $p$ value was determined for those who did and did not experience local failure. Patients with lesions treated with a dose above the cut point were designated as the high-dose cohort, and those given less than the cut-point dose were designated as the lowdose cohort.

\section{Results}

The mean overall survival for the entire cohort was 26.9 months (range $2-141$ months). The mean prescribed dose was 2400 cGy (range 1600-2600 cGy). The median GTV, GTV D95, and GTV V95 were $13.4 \mathrm{~cm}^{3}$ (range $0.13-642 \mathrm{~cm}^{3}$ ), $2320 \mathrm{cGy}$ (range 1334-2850 cGy), and 98\% (range 69\%-100\%), respectively. The median PTV, PTV D95, and PTV V95 were found to be $65.9 \mathrm{~cm}^{3}$ (range 4-904 cm $\mathrm{cm}^{3}$ ), $2205 \mathrm{cGy}$ (range 1414-2864 cGy), and 94.8\% (range 80\%-100\%), respectively. The most common histological types were renal cell carcinoma $(n=170)$, sarcoma $(\mathrm{n}=113)$, non-small cell lung carcinoma $(\mathrm{n}=102)$, pros- tate cancer $(n=91)$, and colorectal cancer $(n=71)$. Tumor characteristics are summarized in Table 1.

A total of 28 lesions have progressed. The mean time to failure was 26 months (range 9.7-57 months). There was no significant difference in the GTV (median $38 \mathrm{~cm}^{3}$ vs 26 $\mathrm{cm}^{3}$ ) and PTV (median $73 \mathrm{~cm}^{3}$ vs $65 \mathrm{~cm}^{3}$ ) for lesions that achieved local control and those that did not. The GTV V95 (median 95\% vs 98\%) and PTV V95 (median 93\% vs $95 \%$ ) were similar for patients who experienced local failure or whose lesion remained controlled, which suggests that patients had equal dose coverage of the GTV and PTV relative to the intended dose and equally highquality treatment plans regardless of whether the treatment ultimately failed or succeeded.

However, absolute dose parameters demonstrated significant differences between controlled and uncontrolled cases. We examined the 10th-90th percentile of GTV D95 and PTV D95. The optimal dose cutoff in terms of time to local failure was found to be 1830 cGy for GTV D95 and 1740 cGy for PTV D95 (Figs. 1 and 2), which after adjustment for multiple testing still yielded a highly significant result based on the maximal chi-square method $(\mathrm{p}<0.001)$. When defined by these dose cut points, the high-dose GTV and PTV D95 cohorts had a 2.5\% and $2.3 \%$ overall crude rate of local progression, respectively, and the low-dose GTV and PTV D95 cohorts experienced 


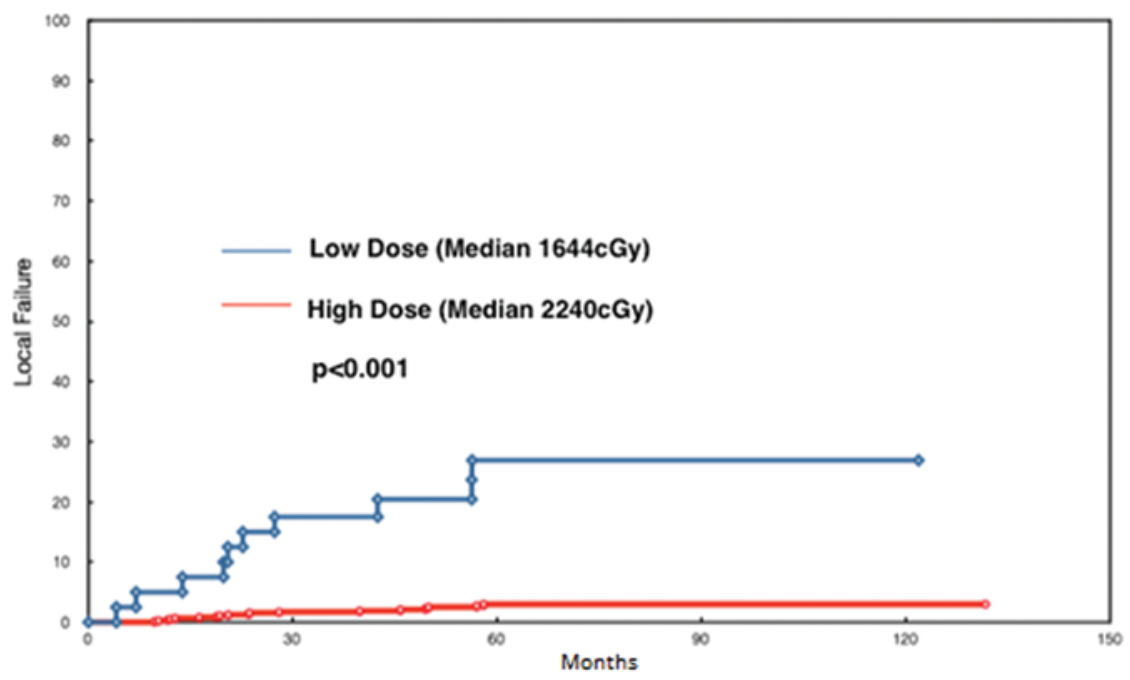

\begin{tabular}{|l|l|l|l|l|}
\hline \multirow{2}{*}{ CIF\% (95\%CI) } & 12 months & 24 months & 36 months & 48 months \\
\hline \multirow{3}{*}{ Overall } & $0.63 \%(0.08-1.2 \%)$ & $2.3 \%(1.2-3.3 \%)$ & $2.6 \%(1.2-3.7 \%)$ & $3.1 \%(1.8-4.4 \%)$ \\
\cline { 2 - 5 } PTV D95 Low Dose & $5 \%(0-12 \%)$ & $15 \%(3.7-26 \%)$ & $18 \%(5.5-30 \%)$ & $20 \%(7.4-33 \%)$ \\
\cline { 2 - 5 } & & & & \\
PTV D95 High Dose & $0.41 \%(0-0.87 \%)$ & $1.6 \%(0.65-2.5 \%)$ & $1.7 \%(0.75-2.7 \%)$ & $2.1 \%(1-3.2 \%)$ \\
\hline
\end{tabular}

FIG. 2. Upper: Local control for PTV D95 cut point of 1740 cGy. The high-dose cohort median dose is $2240 \mathrm{cGy}$, and the low-dose cohort median dose is $1644 \mathrm{cGy}$. Lower: Chart showing the CIF.

a $14.5 \%$ and $25 \%$ overall crude rate of progression, respectively. Analyzed with these cut points, the median GTV D95 for the high-dose cohort was $2356 \mathrm{cGy}$, and it was 1709 cGy for the low-dose group. The median PTV D95 values for the high- and low-dose cohorts were 2240 cGy and 1644 cGy, respectively (Table 2).

The cumulative incidence of local failure (CIF) for the entire group was $3.1 \%$ at 48 months. When analyzed by the absolute dose given, the CIF for the high-dose GTV D95 and PTV D95 cohorts at 48 months was found to be 2.1\% (Fig. 1). The low-dose GTV D95 and PTV D95 cohorts demonstrated a significantly worse rate of $14 \%$ and $20 \%$ CIF, respectively, at the same 48-month time point ( $\mathrm{p}$ $<0.001$ ) (Fig. 2).

Histologically, local control varied from $80 \%$ (breast cancer with one local failure at 57 months) to $100 \%$ (cervical cancer and hepatobiliary cancer). The outcomes by histological type are summarized in Table 1. Both GTV D95 and PTV D95 were highly significantly associated with local failure in univariate analysis ( $p<0.001)$, but tumor histological type and GTV and PTV or GTV and PTV V95 values had no significant impact on the rate of local failure (Table 3).

In this series, 2 cases of myelopathy were observed out of $476(0.42 \%)$ cervical and thoracic levels treated. Both cases were observed in the absence of documented tumor progression. In each case, the maximum spinal cord dose did not exceed $1400 \mathrm{cGy}$. According to the Radiation Therapy Oncology Group/European Organization for Research and Treatment of Cancer (RTOG/EORTC) late radiation morbidity scoring system for the spinal cord, both sustained Grade 3 toxicity, defined as objective neurological findings at or below the cord level treated. ${ }^{24}$

\section{Discussion}

This analysis was performed in every patient treated at a single institution whose records were obtained from a prospectively maintained database. During the entire period covered by this report, the same core multidisciplinary team, treatment philosophy, and relatively uniform treatment techniques and radiation doses were involved with each treatment. Therefore, there has been relatively little variation in how patients in this database were initially assessed, selected, treated, and then re-assessed in follow-up.

Because interinstitutional prescribing practices may vary (for example, 1800 cGy prescribed to the $80 \%$ isodose line will produce very similar D95 values to 2400 cGy prescribed to $100 \%$ ), this analysis was performed on not just the prescribed dose but on the actual absolute dose. This study demonstrates the importance of adequate delivery of the absolute dose to the GTV (D95 median 2352 cGy, 1830 cGy minimum) and PTV (D95 median 2232 cGy, 1740 cGy minimum) to achieve durable tumor control with a $2 \%$ risk of local failure. When this can be achieved, other characteristics such as tumor histological type and size are no longer significant factors for durable local control of spinal metastases. At our institution, these doses are achieved when $2400 \mathrm{cGy}$ is prescribed to the normalized $100 \%$ isodose line. Also, our experience has demonstrated that the requisite high doses needed for excellent tumor control are achievable in almost all cases. 


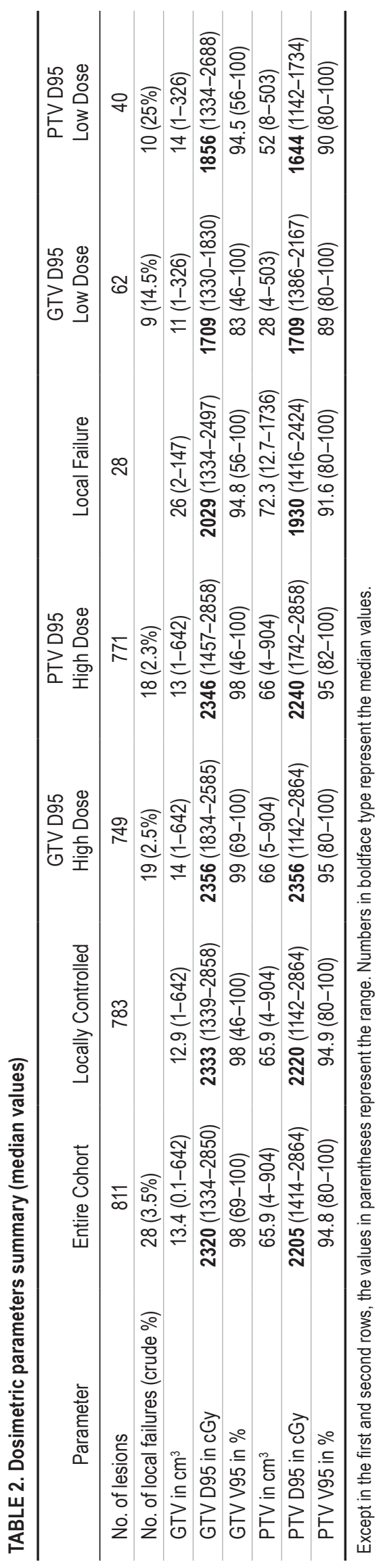

TABLE 3. Univariate analysis for local control

\begin{tabular}{lcc}
\hline \multicolumn{1}{c}{ Factor } & Hazard Ratio & p Value \\
\hline PTV (continuous, divided by 100) & 1.14 & 0.25 \\
\hline GTV (continuous, divided by 100) & 1.30 & 0.06 \\
\hline PTV D95 (continuous, Gy) & 0.70 & $<0.001$ \\
\hline GTV D95 (continuous, Gy) & 0.77 & $<0.001$ \\
\hline PTV V95 (continuous) & 0.96 & $<0.25$ \\
\hline GTV V95 (continuous) & 0.97 & 0.13 \\
\hline $\begin{array}{c}\text { Histological findings (radiosensitive } \\
\text { vs radioresistant) }\end{array}$ & 1.44 & 0.40 \\
\hline
\end{tabular}

Although others have reported excellent outcomes with spine radiosurgery for traditionally radioresistant histological types such as renal cell carcinoma, ${ }^{9,29}$ sarcoma, ${ }^{7}$ and melanoma ${ }^{10}$ little detailed dosimetric information, apart from the prescribed dose, has been analyzed in conjunction with histological findings in assessing local control. The most unique feature of spine radiosurgery is the ability to deliver an ablative dose of radiation to the tumor in a single fraction-hence, details concerning the actual dose, rather than the intended dose, and its relationship to durable tumor control are important to note. The dose of radiation is associated with antitumor cell effects such as DNA double-strand breaks, but may also reflect synergistic mechanisms of action unique to high-dose-per-fraction treatment, such as tumor vascular endothelial damage, ${ }^{8,21}$ and possibly immunological effects in the presence of immune-modulating drug therapy. ${ }^{20,25}$ These cytotoxic mechanisms have been demonstrated and described both preclinically and clinically.

Tumor histological type was not found to be associated with the probability of local failure, a finding that is in stark contrast to reported outcomes with conventionally fractionated palliative spine radiotherapy, ${ }_{11}^{11}$ where metastases from radioresistant histological types do significantly worse than more radiosensitive histological types such as breast cancer and prostate cancer. ${ }^{13,16,17}$ Therefore, SRS should be considered in particular for resistant histological types, because the outcomes for spine radiosurgery are equally good, regardless of histological phenotype, when a sufficient dose is delivered.

Durability of response is also a unique aspect of spine radiosurgery when compared with conventionally fractionated, palliative spine radiotherapy. Large retrospective analyses of spinal metastases suggest that for unfavorable histological types the rate of relapse approaches $80 \%$ within 2 years,,$^{13,17}$ and the rate of failure continues to increase with longer follow-up. A small randomized trial of surgical decompression for spinal cord compression followed by conventional palliative radiation therapy reported that only $25 \%$ of patients remained ambulatory at 1 year posttreatment, and less than $10 \%$ remained ambulatory after 2 years. ${ }^{19}$ Randomized trials of different conventional fractionation schedules for treating bone metastases, the majority of which were spine, have reported acceptable outcomes at 3-4 months; however, longer follow-up at 1 year demonstrates that the rates of palliative failure essentially double. ${ }^{26,28}$ Given the extended survival of patients 
with spinal metastases resulting from increasingly effective systemic agents, a 3- to 4-month outcome is no longer clinically relevant. Patients need a more durable treatment for the optimal management of spinal metastases. The outcomes of this report, with the longest follow-up now extending beyond 10 years, suggest that high-dose, singlefraction spine radiosurgery is capable of providing durable tumor control, with a better than $20 \%$ risk of local relapse at lower doses and a $2 \%$ risk of progression at a higher dose out to 4 years. This response is independent of histological type, which is consistent with the literature. .,2,18 $^{1}$

A detailed analysis of toxicity is beyond the scope of this study, but we have reported extensively on the risks of vertebral body fracture, ${ }^{22}$ esophageal complications, ${ }^{3}$ and neuropathy. ${ }^{27}$ Although symptomatic fractures are the most common event ( $12 \%$ require interventions $)^{18}$ and are easily palliated, severe neuropathy and esophageal complications are also possible, but fortunately are very rare. Regarding a maximal spinal cord dose constraint of 1400 cGy, an acceptably low rate $(0.42 \%)$ of myelopathy was found in the current study.

\section{Conclusions}

A detailed dosimetric analysis of the largest reported single-institution experience of spine radiosurgery has demonstrated that this is an effective treatment for solid tumors that are metastatic to the spine. Durable local control has been achieved regardless of tumor phenotype or size when doses are in the range of a GTV D95 of 2395 cGy and PTV D95 of 2232 cGy, resulting in a CIF of only $2.1 \%$ at 48 months. In particular, spine radiosurgery should be considered for patients with a good prognosis in whom long-term local control is required for radioresistant disease.

\section{Acknowledgments}

This work was supported in part by NIH/NCI core grant P30 CA008748.

\section{References}

1. Bate BG, Khan NR, Kimball BY, Gabrick K, Weaver J: Stereotactic radiosurgery for spinal metastases with or without separation surgery. J Neurosurg Spine 22:409-415, 2015

2. Chan NK, Abdullah KG, Lubelski D, Steinmetz MP, Benzel EC, Shin JH, et al: Stereotactic radiosurgery for metastatic spine tumors. J Neurosurg Sci 58:37-44, 2014

3. Cox BW, Jackson A, Hunt M, Bilsky M, Yamada Y: Esophageal toxicity from high-dose, single-fraction paraspinal stereotactic radiosurgery. Int J Radiat Oncol Biol Phys 83:e661-e667, 2012

4. Cox BW, Spratt DE, Lovelock M, Bilsky MH, Lis E, Ryu S, et al: International Spine Radiosurgery Consortium consensus guidelines for target volume definition in spinal stereotactic radiosurgery. Int J Radiat Oncol Biol Phys 83:e597e605, 2012

5. de Moraes FY, Taunk NK, Laufer I, Neves-Junior WFP, Hanna SA, de Andrade Carvalho $\mathrm{H}$, et al: Spine radiosurgery for the local treatment of spine metastases: Intensity-modulated radiotherapy, image guidance, clinical aspects and future directions. Clinics (Sao Paulo) 71:101-109, 2016
6. Fine JP, Gray RJ: A proportional hazards model for the subdistribution of a competing risk. J Am Stat Assoc 94:496509, 1999

7. Folkert MR, Bilsky MH, Tom AK, Oh JH, Alektiar KM, Laufer I, et al: Outcomes and toxicity for hypofractionated and single-fraction image-guided stereotactic radiosurgery for sarcomas metastasizing to the spine. Int J Radiat Oncol Biol Phys 88:1085-1091, 2014

8. Garcia-Barros M, Paris F, Cordon-Cardo C, Lyden D, Rafii S, Haimovitz-Friedman A, et al: Tumor response to radiotherapy regulated by endothelial cell apoptosis. Science 300:1155-1159, 2003

9. Gerszten PC, Burton SA, Ozhasoglu C, Vogel WJ, Welch WC, Baar J, et al: Stereotactic radiosurgery for spinal metastases from renal cell carcinoma. J Neurosurg Spine 3:288295, 2005

10. Gerszten PC, Burton SA, Quinn AE, Agarwala SS, Kirkwood JM: Radiosurgery for the treatment of spinal melanoma metastases. Stereotact Funct Neurosurg 83:213-221, 2005

11. Gerszten PC, Mendel E, Yamada Y: Radiotherapy and radiosurgery for metastatic spine disease: what are the options, indications, and outcomes? Spine (Phila Pa 1976) 34 (22 Suppl):S78-S92, 2009

12. Gray R: A class of K-sample tests for comparing the cumulative incidence of a competing risk. Ann Stat 16:1141-1154, 1988

13. Katagiri H, Takahashi M, Inagaki J, Kobayashi H, Sugiura $\mathrm{H}$, Yamamura S, et al: Clinical results of nonsurgical treatment for spinal metastases. Int J Radiat Oncol Biol Phys 42:1127-1132, 1998

14. Laufer I, Rubin DG, Lis E, Cox BW, Stubblefield MD, Yamada Y, et al: The NOMS framework: approach to the treatment of spinal metastatic tumors. Oncologist 18:744-751, 2013

15. Lovelock DM, Hua C, Wang P, Hunt M, Fournier-Bidoz N, Yenice K, et al: Accurate setup of paraspinal patients using a noninvasive patient immobilization cradle and portal imaging. Med Phys 32:2606-2614, 2005

16. Maranzano E, Bellavita R, Rossi R, De Angelis V, Frattegiani A, Bagnoli R, et al: Short-course versus split-course radiotherapy in metastatic spinal cord compression: results of a phase III, randomized, multicenter trial. J Clin Oncol 23:3358-3365, 2005

17. Mizumoto M, Harada H, Asakura H, Hashimoto T, Furutani $\mathrm{K}$, Hashii H, et al: Radiotherapy for patients with metastases to the spinal column: a review of 603 patients at Shizuoka Cancer Center Hospital. Int J Radiat Oncol Biol Phys 79:208-213, 2011

18. Moussazadeh N, Lis E, Katsoulakis E, Kahn S, Svoboda M, DiStefano NM, et al: Five-year outcomes of high-dose singlefraction spinal stereotactic radiosurgery. Int J Radiat Oncol Biol Phys 93:361-367, 2015

19. Patchell RA, Tibbs PA, Regine WF, Payne R, Saris S, Kryscio RJ, et al: Direct decompressive surgical resection in the treatment of spinal cord compression caused by metastatic cancer: a randomised trial. Lancet 366:643-648, 2005

20. Postow MA, Callahan MK, Barker CA, Yamada Y, Yuan J, Kitano S, et al: Immunologic correlates of the abscopal effect in a patient with melanoma. N Engl J Med 366:925-931, 2012

21. Rao SS, Thompson C, Cheng J, Haimovitz-Friedman A, Powell SN, Fuks Z, et al: Axitinib sensitization of high single dose radiotherapy. Radiother Oncol 111:88-93, 2014

22. Rose PS, Laufer I, Boland PJ, Hanover A, Bilsky MH, Yamada J, et al: Risk of fracture after single fraction image-guided intensity-modulated radiation therapy to spinal metastases. J Clin Oncol 27:5075-5079, 2009

23. Sahgal A, Bilsky M, Chang EL, Ma L, Yamada Y, Rhines LD, et al: Stereotactic body radiotherapy for spinal metasta- 
ses: current status, with a focus on its application in the postoperative patient. J Neurosurg Spine 14:151-166, 2011

24. Sahgal A, Ma L, Gibbs I, Gerszten PC, Ryu S, Soltys S, et al: Spinal cord tolerance for stereotactic body radiotherapy. Int J Radiat Oncol Biol Phys 77:548-553, 2010

25. Seung SK, Curti BD, Crittenden M, Walker E, Coffey T, Siebert JC, et al: Phase 1 study of stereotactic body radiotherapy and interleukin-2-tumor and immunological responses. Sci Transl Med 4:137ra74, 2012

26. Steenland E, Leer JW, van Houwelingen H, Post WJ, van den Hout WB, Kievit J, et al: The effect of a single fraction compared to multiple fractions on painful bone metastases: a global analysis of the Dutch Bone Metastasis Study. Radiother Oncol 52:101-109, 1999 (Erratum in Radiother Oncol 53:167, 1999)

27. Yamada Y, Bilsky MH, Laufer I, Ruppert L, Joan Z, McBride $\mathrm{S}$, et al: Radiculopathy or plexopathy associated with high dose stereotactic radiosurgery of spinal metastasis. Int J Radiat Oncol Biol Phys 90 (1 Suppl):S125, 2014 (Abstract \#272)

28. Yarnold JR: 8 Gy single fraction radiotherapy for the treatment of metastatic skeletal pain: randomised comparison with a multifraction schedule over 12 months of patient follow-up. Radiother Oncol 52:111-121, 1999

29. Zelefsky MJ, Greco C, Motzer R, Magsanoc JM, Pei X, Lovelock M, et al: Tumor control outcomes after hypofractionated and single-dose stereotactic image-guided intensity-modulated radiotherapy for extracranial metastases from renal cell carcinoma. Int J Radiat Oncol Biol Phys 82:1744-1748, 2012

\section{Disclosures}

Dr. Laufer is a consultant for Globus, SpineWave, and DePuy/ Synthes. Dr. Lis is a consultant for Medtronic. Dr. Yamada is a consultant for Varian Medical Systems, and he is a member of the medical advisory board for the Chordoma Foundation.

\section{Author Contributions}

Conception and design: Yamada, Katsoulakis, Schmitt, Higginson, Zelefsky, Bilsky. Acquisition of data: Yamada, Katsoulakis, Barzilai, Lis, Mechalakos. Analysis and interpretation of data: Yamada, Lovelock, Barzilai, Lis, Mechalakos. Drafting the article: Yamada, Bilsky. Critically revising the article: Yamada, Laufer, Bilsky. Reviewed submitted version of manuscript: Yamada, Katsoulakis, Laufer, Barzilai, McLaughlin, Zhang, Schmitt, Higginson, Lis, Bilsky. Approved the final version of the manuscript on behalf of all authors: Yamada. Statistical analysis: Yamada, Zhang. Administrative/technical/material support: McLaughlin, Zelefsky. Study supervision: Yamada.

\section{Supplemental Information Videos \\ Video abstract. https://vimeo.com/194713079.}

\section{Correspondence}

Yoshiya Yamada, Department of Radiation Oncology, Memorial Sloan Kettering Cancer Center, 1275 York Ave., New York, NY 10065. email: yamadaj@mskcc.org. 